\title{
Analysis of a tunable coupler for superconducting phase qubits
}

\author{
Ricardo A. Pinto and Alexander N. Korotkov* \\ Department of Electrical Engineering, University of California, Riverside, California 92521, USA \\ Michael R. Geller \\ Department of Physics and Astronomy, University of Georgia, Athens, Georgia 30602, USA \\ Vitaly S. Shumeiko \\ Department of Microtechnology and Nanoscience, MC2, Chalmers University of Technology, SE-41296 Gothenburg, Sweden
}

John M. Martinis

Department of Physics, University of California, Santa Barbara, California 93106, USA

(Received 8 July 2010; published 28 September 2010)

\begin{abstract}
This paper presents a theoretical analysis of the recently realized tunable coupler for superconducting phase qubits [R. C. Bialczak et al., arXiv:1007.2219 (unpublished)]. The coupling can be turned off by compensating a negative mutual inductance with a tunable Josephson inductance. The main coupling in this system is of the $X X$ type and can be zeroed exactly, while there is also a small undesired contribution of the $Z Z$ type. We calculate both couplings as functions of the tuning parameter (bias current) and focus on the residual coupling in the OFF regime. In particular, we show that for typical experimental parameters the coupling OFF/ON ratio is few times $10^{-3}$, and it may be zeroed by proper choice of parameters. The remaining errors due to physical presence of the coupler are on the order of $10^{-6}$.
\end{abstract}

DOI: 10.1103/PhysRevB.82.104522

PACS number(s): 03.67.Lx, 85.25.Cp

\section{INTRODUCTION}

Superconducting qubits ${ }^{1}$ are potential building blocks of a quantum computer. Among their advantages in comparison with other qubit types are an efficient control with voltage/ current/microwave pulses and use of a well-developed technology suitable for large-scale integration. Recent demonstrations of simple quantum algorithms ${ }^{2,3}$ and three-qubit entanglement ${ }^{4,5}$ with superconducting qubits are important steps toward a practical quantum computation.

In the standard idea of a gate-based quantum computation $^{6}$ it is important that the qubits are decoupled from each other for most of the time, and the coupling of a desired type between two (or three) qubits is switched on only for a quantum gate operation, while it is switched off again after that. Since the superconducting qubits cannot be physically moved in space, such coupling/decoupling should be realized by changing control parameters of a circuit. The simplest idea is to tune the qubits in resonance with each other for efficient coupling and move them out of resonance for decoupling (see, e.g., Refs. 2-5). However, this requires avoiding unwanted resonances, and with increasing number of qubits may lead to the problem of "spectral crowding." Even more important limitation of this approach is the following. Because the effective coupling strength when the qubits are detuned by an energy $\Delta E$ is of order $g^{2} / \Delta E$, where $g$ is the tuned value, the ratio of the "switched-off" and "switched-on" coupling strengths is $\mathrm{OFF} / \mathrm{ON} \simeq g / \Delta E$. This "OFF/ON ratio" characterizes a coupler's ability to successfully turn on and off the coupling between qubits. $\Delta E / h$ is limited to about a gigahertz in current superconducting qubit devices. To make the ratio small then requires $g$ to be small, which makes gate operations slow. For example, to realize an OFF/ON ratio of $10^{-3}$ when $\Delta E / h=1 \mathrm{GHz}$ would require $g$ to be $1 \mathrm{MHz}$, which is unacceptably small.
A different idea is to introduce an extra element between the qubits: an adjustable coupler, which can turn the coupling on and off. It is a much better approach from the architecture point of view since it allows easier design of complex quantum circuits. This has motivated several experimental ${ }^{7-12}$ and theoretical $^{13-23}$ studies of adjustable couplers for superconducting qubits.

In this paper we theoretically analyze operation of a recently realized ${ }^{12}$ tunable coupler for superconducting phase qubits, which demonstrated current-controlled tuning of the $X X$-type coupling from 0 to $100 \mathrm{MHz}$. In the next section we discuss the Hamiltonian of the analyzed Josephson circuit and our definition of the two-qubit coupling frequencies $\Omega_{X X}$ and $\Omega_{Z Z}$, corresponding to the $X X$ and $Z Z$ types of interaction (for our system typically $\left|\Omega_{X X}\right| \gg\left|\Omega_{Z Z}\right|$, so the main coupling is of the $X X$ type). In Sec. III we find $\Omega_{X X}$ and $\Omega_{Z Z}$ in a simple semiclassical way while in Sec. IV similar results are obtained in the lowest-order quantum analysis. We show that both $\Omega_{X X}$ and $\Omega_{Z Z}$ can cross zero as functions of the control parameter (bias current), but typically not simultaneously, thus leading to a nonvanishing residual coupling, which is discussed in Sec. V. In particular, we show that typical $\mathrm{OFF} / \mathrm{ON}$ ratio for the coupler is few times $10^{-3}$; however, a minor modification of the experimental circuit ${ }^{12}$ (addition of a small coupling capacitance) can zero the residual coupling, thus zeroing the $\mathrm{OFF} / \mathrm{ON}$ ratio. Actually, this does not mean complete decoupling, because we rely on the two-qubit description of a more complicated circuit. The remaining coupling effects are also discussed in Sec. $\mathrm{V}$ and are shown to lead to errors on the order of $10^{-6}$. Section VI presents numerical results of the quantum analysis; for typical experimental parameters they are close to the analytical results. Section VII is the conclusion. In Appendix we discuss the position and momentum matrix elements for an oscillator 


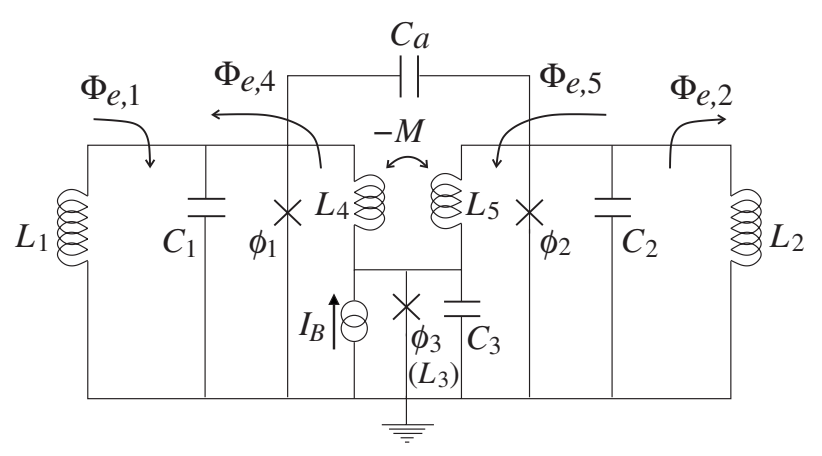

FIG. 1. The analyzed scheme of two-qubit coupling, which is controlled by the bias current $I_{B}$ of the coupling Josephson junction. Indices 1 and 2 refer to the two qubits and index 3 refers to the coupling junction. Coupling inductors $L_{4}$ and $L_{5}$ have a negative mutual inductance $-M$. The current $I_{B}$ controls the Josephson inductance $L_{3}$ of the coupling junction, which effectively adds to $-M$. An additional small coupling is via capacitance $C_{a}$.

with weak cubic nonlinearity and derive improved analytics for $\Omega_{X X}$ and $\Omega_{Z Z \text {. }}$

\section{SYSTEM AND HAMILTONIAN}

Let us consider the system ${ }^{12}$ shown in Fig. 1, which consists of two flux-biased phase qubits ${ }^{24}$ characterized by capacitances $C_{1}$ and $C_{2}$, inductances $L_{1}$ and $L_{2}$, and Josephson energies $E_{J 1}$ and $E_{J 2}$ of the junctions (in Fig. 1 the superconducting phases across these Josephson junctions are denoted as $\phi_{1}$ and $\phi_{2}$ ). The qubits are coupled via an additional Josephson junction (characterized by $C_{3}$ and $E_{J 3}$ ) with an adjustable bias current $I_{B}$; the qubits are connected to this junction via inductances $L_{4}$ and $L_{5}$, which have a negative mutual inductance $-M(M>0)$. We also introduce the qubit coupling via a very small capacitance $C_{a}$, which was not implemented in the experiment, ${ }^{12}$ but may be important in future experiments for turning the coupling off more precisely.

The general idea of this scheme ${ }^{12}$ is that the Josephson inductance $L_{3}$ of the middle junction is essentially in series with the mutual inductance $-M$, and therefore (in absence of $C_{a}$ ) the coupling is expected to be (crudely) proportional to $L_{3}-M$. Then varying $L_{3}$ by varying the bias current $I_{B}$, it is possible to adjust the qubit-qubit coupling strength, which is expected to cross zero when $L_{3} \approx M$.

The Hamiltonian of the system can be derived in the standard way ${ }^{25,26}$ and written in terms of the phases $\phi_{i}(i$ $=1,2,3)$ across the three Josephson junctions and the conjugated momenta $p_{i}\left(\left[\phi_{i}, p_{j}\right]=\imath \hbar \delta_{i j}\right)$, which are the corresponding node charges, multiplied by $\widetilde{\Phi}_{0} \equiv \Phi_{0} / 2 \pi=\hbar / 2 e$

$$
\begin{aligned}
H= & \sum_{i=1}^{3}\left[\frac{p_{i}^{2}}{2 \widetilde{C}_{i} \widetilde{\Phi}_{0}^{2}}-E_{J i} \cos \phi_{i}\right]+\sum_{i=1}^{2} \frac{\widetilde{\Phi}_{0}^{2}}{2 L_{i}}\left(\phi_{i}-\phi_{e, i}\right)^{2}-I_{B} \widetilde{\Phi}_{0} \phi_{3} \\
& +\frac{\widetilde{C}_{a}}{C_{1} C_{2}} \frac{p_{1} p_{2}}{\widetilde{\Phi}_{0}^{2}}+\frac{\widetilde{M}}{\widetilde{L}_{4} \widetilde{L}_{5}} \widetilde{\Phi}_{0}^{2}\left(\phi_{1}-\phi_{3}-\phi_{e, 4}\right)\left(\phi_{2}-\phi_{3}-\phi_{e, 5}\right) \\
& +\frac{\widetilde{\Phi}_{0}^{2}}{2 \widetilde{L}_{4}}\left(\phi_{1}-\phi_{3}-\phi_{e, 4}\right)^{2}+\frac{\widetilde{\Phi}_{0}^{2}}{2 \widetilde{L}_{5}}\left(\phi_{2}-\phi_{3}-\phi_{e, 5}\right)^{2}
\end{aligned}
$$

where $\widetilde{C}_{1}=C_{1}+C_{2} C_{a} /\left(C_{2}+C_{a}\right)$ and $\widetilde{C}_{2}=C_{2}+C_{1} C_{a} /\left(C_{1}+C_{a}\right)$ are the effective qubit capacitances, $\widetilde{C}_{3}=C_{3}$ (introduced for notational convenience), $\phi_{e, 1}$ and $\phi_{e, 2}$ are the external dimensionless qubit fluxes, $\phi_{e, 4}$ and $\phi_{e, 5}$ are the external dimensionless fluxes through the loops containing $L_{4}$ and $L_{5}$ (we will often assume $\left.\phi_{e, 4}=\phi_{e, 5}=0\right), \widetilde{C}_{a}=\left(1 / C_{a}+1 / C_{1}\right.$ $\left.+1 / C_{2}\right)^{-1}$ is the effective coupling capacitance, and renormalized coupling inductances are

$$
\frac{\widetilde{L}_{4}}{L_{4}}=\frac{\tilde{L}_{5}}{L_{5}}=\frac{\tilde{M}}{M}=1-\frac{M^{2}}{L_{4} L_{5}} .
$$

All terms in Eq. (1) have clear physical meaning. ${ }^{27}$ Introducing the shifted variables $\delta \phi_{i}=\phi_{i}-\phi_{i, \mathrm{st}}$, where the set $\left\{\phi_{i, \mathrm{st}}\right\}$ corresponds to the minimum of the potential energy, we rewrite Hamiltonian (1) as

$$
\begin{gathered}
H=H_{1}+H_{2}+H_{3}+H_{\mathrm{int}} \\
H_{i}=\frac{p_{i}^{2}}{2 \widetilde{C}_{i} \widetilde{\Phi}_{0}^{2}}+U_{i}\left(\delta \phi_{i}\right), \quad i=1,2,3 \\
H_{\mathrm{int}}=\frac{\widetilde{C}_{a}}{C_{1} C_{2}} \frac{p_{1} p_{2}}{\widetilde{\Phi}_{0}^{2}}+\widetilde{\Phi}_{0}^{2}\left(\frac{\tilde{M}}{\widetilde{L}_{4} \widetilde{L}_{5}} \delta \phi_{1} \delta \phi_{2}-\frac{1+\tilde{M} / \widetilde{L}_{5}}{\tilde{L}_{4}} \delta \phi_{1} \delta \phi_{3}\right. \\
\left.-\frac{1+\widetilde{M} / \tilde{L}_{4}}{\tilde{L}_{5}} \delta \phi_{2} \delta \phi_{3}\right)
\end{gathered}
$$

where the potentials $U_{i}$ have minima at $\delta \phi_{i}=0$ and the corresponding plasma frequencies $\omega_{i, \mathrm{pl}}=\left(\tilde{L}_{i} \widetilde{C}_{i}\right)^{-1 / 2}$ are governed by the effective inductances

$$
\begin{gathered}
\tilde{L}_{1}=\left[L_{1}^{-1}+\widetilde{L}_{4}^{-1}+\widetilde{\Phi}_{0}^{-2} E_{J 1} \cos \phi_{1, \mathrm{st}}\right]^{-1}, \\
\tilde{L}_{2}=\left[L_{2}^{-1}+\widetilde{L}_{5}^{-1}+\widetilde{\Phi}_{0}^{-2} E_{J 2} \cos \phi_{2, \mathrm{st}}\right]^{-1}, \\
\tilde{L}_{3}=\left[\frac{1}{\widetilde{L}_{4}}+\frac{1}{\widetilde{L}_{5}}+\frac{2 \widetilde{M}}{L_{4} \widetilde{L}_{5}}+\frac{1}{\widetilde{\Phi}_{0}} \sqrt{I_{3, \mathrm{cr}}^{2}-I_{3, \mathrm{st}}^{2}}\right]^{-1},
\end{gathered}
$$

where in the last equation we expressed $E_{J 3} \cos \phi_{3, \mathrm{st}}$ in terms of the corresponding junction current $I_{3, \mathrm{st}} \simeq I_{B}$ and the critical current $I_{3, \mathrm{cr}}=E_{J 3} / \widetilde{\Phi}_{0}$.

The Hamiltonians $H_{1}$ and $H_{2}$ correspond to the separated qubits; in absence of coupling two lowest eigenstates in each of them correspond to the logic states $|0\rangle$ and $|1\rangle$. Notice that because of an anharmonicity of the potentials, the qubit frequencies $\omega_{1}$ and $\omega_{2}$ (defined via energy difference between $|1\rangle$ and $|0\rangle$ for uncoupled qubits) are slightly smaller than the plasma frequencies $\omega_{1, \mathrm{pl}}$ and $\omega_{2, \mathrm{pl}}$. The coupler is characterized by the Hamiltonian $H_{3}$; its similarly defined frequency $\omega_{3}$ is slightly smaller than $\omega_{3, p}$. In the experiment ${ }^{12} \omega_{3}$ was almost an order of magnitude higher than $\omega_{1,2}$, and the coupler had only virtual excitations. In our analysis we also assume absence of real excitations in the coupler; however, in general we do not assume $\omega_{3} \gg \omega_{1,2}$ (except specially mentioned), we only assume absence of resonance between the coupler and the qubits. 
Our goal is to calculate the coupling between the two qubits due to $H_{\text {int }}$ (we assume a weak coupling). In general, the coupling between two logic qubits can be characterized by nine real parameters. However, since for phase qubits the energies of the states $|0\rangle$ and $|1\rangle$ are significantly different, we can use the rotating wave approximation (RWA), i.e., neglect terms creating two excitations or annihilating two excitations; then there are only three coupling parameters in the rotating frame

$$
\begin{aligned}
H_{c}= & \frac{\hbar \Omega_{X X}}{4}\left(\sigma_{X}^{(1)} \sigma_{X}^{(2)}+\sigma_{Y}^{(1)} \sigma_{Y}^{(2)}\right)+\frac{\hbar \Omega_{X Y}}{4}\left(\sigma_{X}^{(1)} \sigma_{Y}^{(2)}-\sigma_{Y}^{(1)} \sigma_{X}^{(2)}\right) \\
& +\frac{\hbar \Omega_{Z Z}}{4} \sigma_{Z}^{(1)} \sigma_{Z}^{(2)},
\end{aligned}
$$

where superscripts of the Pauli matrices indicate qubit numbering. Moreover, from the symmetry of the interaction Hamiltonian (5) it follows that $\Omega_{X Y}=0$ (because matrix elements of $\delta \phi_{i}$ are real, and for $p_{i}$ they are imaginary); therefore our goal is to calculate only two coupling frequencies: $\Omega_{X X}$ and $\Omega_{Z Z}$. Notice that since the $X X$ and $Y Y$ interactions are indistinguishable in the RWA (both correspond to $\left.\sigma_{+}^{(1)} \sigma_{-}^{(2)}+\sigma_{-}^{(1)} \sigma_{+}^{(2)}\right)$, we may rewrite the first term in Eq. (9) as $\left(\hbar \Omega_{X X} / 2\right) \sigma_{X}^{(1)} \sigma_{X}^{(2)}$.

The considered system of Fig. 1 has a large Hilbert space, which can, in principle, be reduced to a two-qubit space in a variety of ways, giving, in general, different values of $\Omega_{X X}$ and $\Omega_{Z Z}$. To avoid ambiguity, we define the coupling frequencies in terms of the exact eigenstates of the full physical system. In particular, we associate the two-qubit logic states $|00\rangle$ and $|11\rangle$ with the corresponding eigenstates of the full Hamiltonian (3) and denote their energies as $E_{00}$ and $E_{11}$; for this association we start with the product state using the ground state for the coupler, and then find the nearest eigenstate. Similarly, instead of trying to define the uncoupled logic states $|01\rangle$ and $|10\rangle$ (that is ambiguous, though a natural definition can be based on the dressed states discussed in Sec. IV), we deal with the eigenstates resulting from their coupling, which are associated with two exact eigenstates of the full system; their energies are denoted as $E_{+}$and $E_{-}$ $\left(E_{+} \geq E_{-}\right)$.

Then the coupling $\Omega_{Z Z}$ is defined as

$$
\hbar \Omega_{Z Z}=E_{00}+E_{11}-\left(E_{+}+E_{-}\right),
$$

which is obviously consistent with Eq. (9) for logic qubits (a similar definition has been used in Ref. 2). The coupling $\Omega_{X X}$ can be defined as the minimal splitting in the avoided level crossing between $|01\rangle$ and $|10\rangle$, i.e., as

$$
\left|\hbar \Omega_{X X}\right|=\min _{\omega_{1}-\omega_{2}}\left(E_{+}-E_{-}\right)
$$

with the sign of $\Omega_{X X}$ easily obtained by comparing with Eq. (9). Actually, the definition in Eq. (11) cannot be applied to an arbitrary qubit detuning $\omega_{1}-\omega_{2}$ (in a symmetric case it works only for degenerate qubits); such generalization of $\Omega_{X X}$ definition can be done by comparing exact eigenstates with the standard avoided level-crossing behavior (discussed in more detail in Secs. IV and V). Notice that our definitions of $\Omega_{X X}$ and $\Omega_{Z Z}$ do not need any assumption of a weak coupling (this is their main advantage); however, a weak coupling will be assumed in derivation of analytical results.

\section{SIMPLE SEMICLASSICAL ANALYSIS}

Let us first calculate $\Omega_{X X}$ and $\Omega_{Z Z}$ in a simple, essentially electrical engineering way (we will see later that the result is close to the quantum result). For simplicity in this section we assume $\left(L_{4}, L_{5}\right) \gg\left(\tilde{L}_{1}, \tilde{L}_{2}, \widetilde{L}_{3}, M\right), C_{a} \ll\left(C_{1}, C_{2}\right)$, so that the coupling is weak and the tilde signs in many cases can be avoided. We also replace the middle junction with the effective inductance $L_{3}=\widetilde{\Phi}_{0} / \sqrt{I_{3, \mathrm{cr}}^{2}-I_{3, \mathrm{st}}^{2}} \approx \widetilde{\Phi}_{0} / \sqrt{I_{3, \mathrm{cr}}^{2}-I_{B}^{2}}$ (in this approximation $\tilde{L}_{3} \approx L_{3}$ ).

The coupling $\Omega_{X X}$ corresponds to the frequency splitting between the symmetric and antisymmetric modes of the twoqubit oscillations. So, let us assume degenerate qubits, $\omega_{1}$ $=\omega_{2}=\omega_{\mathrm{qb}}$, and find the splitting in the classical linear system. Notice that at frequency $\omega_{\mathrm{qb}}$ the capacitance $C_{3}$ is equivalent to the inductance $-1 /\left(\omega_{\mathrm{qb}}^{2} C_{3}\right)$, and therefore the parallel connection of $L_{3}$ and $C_{3}$ is equivalent to the inductance

$$
L_{3}^{\mathrm{eff}}=\frac{L_{3}}{1-\omega_{\mathrm{qb}}^{2} L_{3} C_{3}}=\frac{L_{3}}{1-\left(\omega_{\mathrm{qb}} / \omega_{3}\right)^{2}},
$$

notice that here $\omega_{3}=\omega_{3, \mathrm{pl}}$ for the coupler since we assume a linear system.

Suppose $\omega \approx \omega_{\mathrm{qb}}$ is a classical eigenfrequency and the first qubit voltage is $V_{1} e^{i \omega t}$. Then using the phasor representation, we find the current through $L_{4}$ as $I_{4}=V_{1} /\left(i \omega L_{4}\right)$; it induces the voltage $V_{\mathrm{cp}}=i \omega\left(L_{3}^{\mathrm{eff}}-M\right) I_{4}$ in the coupling inductances, which causes the current $I_{5}=V_{\mathrm{cp}} /\left(i \omega L_{5}\right)=V_{1}\left(L_{3}^{\mathrm{eff}}\right.$ $-M) /\left(i \omega L_{4} L_{5}\right)$ flowing through $L_{5}$ into the second qubit. Adding this current to the current $I_{a}=i \omega C_{a} V_{1}$ through $C_{a}$, we get the total current $I_{2}=I_{5}+I_{a}$, flowing into the second qubit. The extra current $I_{2}$ is equivalent to changing the qubit capacitance $C_{2}$ by $\Delta C_{2}=-I_{2} /\left(i \omega V_{2}\right)$, where $V_{2}= \pm V_{1} \sqrt{C_{1} / C_{2}}$ is the second qubit voltage for the symmetric and antisymmetric modes (the factor $\sqrt{C_{1} / C_{2}}$ comes from the condition of equal energies in the two qubits). The effective change in the capacitance slightly changes the oscillation frequency $\left(\tilde{L}_{2} C_{2}\right)^{-1 / 2}$ so the eigenfrequency can be found as $\omega=\omega_{\mathrm{qb}}(1$ $\left.-\Delta C_{2} / 2 C_{2}\right)$. Therefore, the frequency splitting due to coupling is $\left|\Omega_{X X}\right|=\omega_{\mathrm{qb}}\left|\Delta C_{2}\right| / C_{2}$, and substituting $\Delta C_{2}$ we finally find

$$
\Omega_{X X}=\frac{M-L_{3} /\left[1-\left(\omega_{\mathrm{qb}} / \omega_{3}\right)^{2}\right]}{L_{4} L_{5} \omega_{\mathrm{qb}} \sqrt{C_{1} C_{2}}}+\frac{C_{a} \omega_{\mathrm{qb}}}{\sqrt{C_{1} C_{2}}},
$$

where the explicit expression in Eq. (12) for $L_{3}^{\text {eff }}$ has been used and the sign of $\Omega_{X X}$ is determined by noticing that a positive $\Omega_{X X}$ should make the frequency (energy) of the symmetric mode larger than for the antisymmetric mode [see Eq. (9)].

The most important observation is that $\Omega_{X X}$ depends on the bias current $I_{B}$, which changes $L_{3}$, and for a proper biasing the coupling $\Omega_{X X}$ can be zeroed exactly. If the correction due to the $C_{a}$ term is small and also $\omega_{\mathrm{qb}} / \omega_{3} \ll 1$ (as in the experiment ${ }^{12}$ ), then $\Omega_{X X}$ is zeroed when $L_{3} \approx M$.

The coupling $\hbar \Omega_{Z Z} \sigma_{Z}^{(1)} \sigma_{Z}^{(2)} / 4$ in Eq. (9) originates from anharmonicity of the qubit potentials and corresponding dif- 
ference between the average Josephson phases for states $|1\rangle$ and $|0\rangle$, which we denote as $\Delta \phi_{10}^{(i)}$ for the $i$ th qubit. This leads to the extra dc current $\widetilde{\Phi}_{0} \Delta \phi_{10}^{(1)} / L_{4}$ through the inductance $L_{4}$, when the first qubit changes state from $|0\rangle$ and $|1\rangle$ and similar dc current change $\widetilde{\Phi}_{0} \Delta \phi_{10}^{(2)} / L_{5}$ through $L_{5}$ for the second qubit. As a result, the state $|11\rangle$ acquires an additional magnetic interaction energy, which is the product of these two currents multiplied ${ }^{27}$ by $M-L_{3}$. This corresponds to

$$
\Omega_{Z Z}=\Delta \phi_{10}^{(1)} \Delta \phi_{10}^{(2)} \frac{\widetilde{\Phi}_{0}^{2}}{\hbar} \frac{M-L_{3}}{L_{4} L_{5}} .
$$

Comparing this result with Eq. (13) for $\Omega_{X X}$, we see absence of the contribution due to $C_{a}$ (which is small anyway) and a similar proportionality to $M-L_{3}$, though without the correction $1-\left(\omega_{\mathrm{qb}} / \omega_{3}\right)^{2}$. This means that by changing the bias current $I_{B}$ (which affects $L_{3}$ ), the coupling $\Omega_{Z Z}$ can be zeroed, and this happens close to the point where $\Omega_{X X}$ is zeroed. Except for the vicinity of the crossing point, $\left|\Omega_{Z Z} / \Omega_{X X}\right| \ll 1$ because $\Delta \phi_{10}^{(i)}$ is small (compared to the ground-state width) for a weak anharmonicity; therefore $\Omega_{X X}$ is the main coupling in our system.

\section{QUANTUM ANALYSIS (ANALYTICS)}

For the quantum analysis let us rewrite the interaction Hamiltonian (5) as

$$
\begin{aligned}
H_{\mathrm{int}}= & K_{13}\left(a_{1}+a_{1}^{\dagger}\right)\left(a_{3}+a_{3}^{\dagger}\right)+K_{23}\left(a_{2}+a_{2}^{\dagger}\right)\left(a_{3}+a_{3}^{\dagger}\right) \\
& +K_{12}\left(a_{1}+a_{1}^{\dagger}\right)\left(a_{2}+a_{2}^{\dagger}\right)+K_{12}^{a}\left(a_{1}-a_{1}^{\dagger}\right)\left(a_{2}-a_{2}^{\dagger}\right),
\end{aligned}
$$

where $a_{i}+a_{i}^{\dagger}=\delta \phi_{i} \sqrt{2 m_{i} \omega_{i} / \hbar}, a_{i}-a_{i}^{\dagger}=\imath p_{i} \sqrt{2 / \hbar m_{i} \omega_{i}}$, and

$$
\begin{gathered}
K_{13}=-\frac{1+\tilde{M} / \tilde{L}_{5}}{\tilde{L}_{4}} k_{13}, \quad K_{23}=-\frac{1+\tilde{M} / \tilde{L}_{4}}{\tilde{L}_{5}} k_{23}, \\
K_{12}=\frac{\tilde{M}}{\tilde{L}_{4} \tilde{L}_{5}} k_{12}, \quad K_{12}^{a}=-\frac{\tilde{C}_{a}}{C_{1} C_{2}} \frac{\hbar^{2}}{4 k_{12}}, \\
k_{i j}=\frac{\hbar \widetilde{\Phi}_{0}^{2}}{2 \sqrt{m_{i} \omega_{i} m_{j} \omega_{j}}}, \quad m_{i}=\widetilde{\Phi}_{0}^{2} \widetilde{C}_{i}
\end{gathered}
$$

(we use the creation/annihilation operators $a_{i}^{\dagger}$ and $a_{i}$ only for brevity of notatons; in their normalization we use the frequency $\omega_{i}$ between two lowest eigenstates instead of the plasma frequency).

In order to find $\Omega_{X X}$, we have to solve the Schrödinger equation $H\left|\psi_{ \pm}\right\rangle=E_{ \pm}\left|\psi_{ \pm}\right\rangle$for the two eigenstates $\left|\psi_{ \pm}\right\rangle\left(E_{+}\right.$ $\geq E_{-}$), corresponding to the coupled logic states $|10\rangle$ and $|01\rangle$. The wave function can be written in the product-state basis as $\left|\psi_{ \pm}\right\rangle=\alpha_{ \pm}|100\rangle+\beta_{ \pm},|001\rangle+\cdots$, where in this notation we show the energy levels $\left|n_{1} n_{3} n_{2}\right\rangle$ of the first qubit, the coupling oscillator (in the middle), and the second qubit, and the terms not shown explicitly should be relatively small in the weak-coupling case. Comparing the amplitudes and energies with the standard avoided level crossing behavior, let us define two coupling frequencies, $\Omega_{X X}^{+}$and $\Omega_{X X}^{-}$, as

$$
\Omega_{X X}^{ \pm}=\frac{E_{ \pm}-E_{\mp}}{\hbar} \frac{2 \alpha_{ \pm} / \beta_{ \pm}}{1+\left(\alpha_{ \pm} / \beta_{ \pm}\right)^{2}} .
$$

We have to define two frequencies because in general $\alpha_{+} / \beta_{+} \neq-\beta_{-} / \alpha_{-}$, in contrast to the ideal case of two logic qubits; this is the price to pay when the two-qubit language is applied to a more complicated physical system. However, the difference $\left|\Omega_{X X}^{+}-\Omega_{X X}^{-}\right|$is typically very small; moreover, for degenerate qubits in a symmetric system $\Omega_{X X}^{+}=\Omega_{X X}^{-}$(because then $\left.\left|\alpha_{ \pm} / \beta_{ \pm}\right|=1\right)$ so that we need only a single frequency $\Omega_{X X}$, which in this case coincides with the definition in Eq. (11). We will neglect the difference between $\Omega_{X X}^{ \pm}$and $\Omega_{X X}$ unless specially mentioned (the difference is important in the case of strongly detuned qubits).

For the analysis it is convenient to express a solution of the Schrödinger equation $H|\psi\rangle=E|\psi\rangle$ as $|\psi\rangle=\alpha\left|\psi_{100}^{\mathrm{dr}}\right\rangle$ $+\beta\left|\psi_{001}^{\mathrm{dr}}\right\rangle$, where the dressed states $\left|\psi_{100}^{\mathrm{dr}}\right\rangle$ and $\left|\psi_{001}^{\mathrm{dr}}\right\rangle$ are defined in the following way. The state $\left|\psi_{100}^{\mathrm{dr}}\right\rangle$ expanded in the product-state basis has the contribution from the state $|100\rangle$ with amplitude 1 and zero contribution from the state $|001\rangle$, i.e., $\left\langle\psi_{100}^{\mathrm{dr}} \mid 100\right\rangle=1$ and $\left\langle\psi_{100}^{\mathrm{dr}} \mid 001\right\rangle=0$. Also, $\left|\psi_{100}^{\mathrm{dr}}\right\rangle$ satisfies equation $\left\langle n|H| \psi_{100}^{\mathrm{dr}}\right\rangle=E\left\langle n \mid \psi_{100}^{\mathrm{dr}}\right\rangle$ for all basis elements $|n\rangle$ $\equiv\left|n_{1} n_{3} n_{2}\right\rangle$ except $|100\rangle$ and $|001\rangle$. The dressed state $\left|\psi_{001}^{\mathrm{dr}}\right\rangle$ is defined similarly, except now $\left\langle\psi_{001}^{\mathrm{dr}} \mid 100\right\rangle=0$ and $\left\langle\psi_{001}^{\mathrm{dr}} \mid 001\right\rangle$ $=1$. Notice that a dressed state is not a solution of an eigenvalue problem; for a given energy $E$ it is a solution of an inhomogeneous systems of linear equations. Also notice that we do not need to normalize the wave functions.

Constructing the dressed states in this way, we have to satisfy (self-consistently for $E$ ) only two remaining equations to solve the Schrödinger equation

$$
\begin{aligned}
& \alpha\left\langle 100|H| \psi_{100}^{\mathrm{dr}}\right\rangle+\beta\left\langle 100|H| \psi_{001}^{\mathrm{dr}}\right\rangle=E \alpha, \\
& \alpha\left\langle 001|H| \psi_{100}^{\mathrm{dr}}\right\rangle+\beta\left\langle 001|H| \psi_{001}^{\mathrm{dr}}\right\rangle=E \beta .
\end{aligned}
$$

Using linear algebra it is easy to prove the reciprocity relation $\left\langle 100|H| \psi_{001}^{\mathrm{dr}}\right\rangle=\left\langle 001|H| \psi_{100}^{\mathrm{dr}}\right\rangle^{*}$ (in our case the complex conjugation is actually not needed since the matrix elements are real), and therefore Eqs. (20) and (21) are similar to the standard equations for an avoided level crossing. Hence, the matrix elements $E_{100}^{\mathrm{dr}}=\left\langle 100|H| \psi_{100}^{\mathrm{dr}}\right\rangle$ and $E_{001}^{\mathrm{dr}}=\left\langle 001|H| \psi_{001}^{\mathrm{dr}}\right\rangle$ play the role of renormalized self-energies of the two-qubit logic states $|10\rangle$ and $|01\rangle$ while the two-qubit coupling can be calculated as

$$
\Omega_{X X}=\frac{2}{\hbar}\left\langle 001\left|H_{\mathrm{int}}\right| \psi_{100}^{\mathrm{dr}}\right\rangle=\frac{2}{\hbar}\left\langle 100\left|H_{\mathrm{int}}\right| \psi_{001}^{\mathrm{dr}}\right\rangle
$$

so that the eigenenergies are given by the usual formula $E_{ \pm}=\left[E_{100}^{\mathrm{dr}}+E_{001}^{\mathrm{dr}} \pm \sqrt{\left(E_{100}^{\mathrm{dr}}-E_{001}^{\mathrm{dr}}\right)^{2}+\hbar^{2} \Omega_{X X}^{2}}\right] / 2[$ in Eq. (22) we wrote $H_{\text {int }}$ instead of $H$ because there is obviously no contribution from the noninteracting part]. Notice that the dressed states and therefore the matrix elements depend on energy $E$, in contrast to the standard level crossing. This leads to a slight difference of $\Omega_{X X}$ for the eigenstates $E_{+}$and $E_{-}$and also makes calculations using Eq. (22) slightly different from the definition in Eq. (19). 
To find $\Omega_{X X}$ analytically, let us assume that the three oscillators are linear, $H_{i}=\hbar \omega_{i}\left(a_{i}^{\dagger} a_{i}+1 / 2\right)$, and use the lowestorder perturbation theory. In the first order

$$
\begin{aligned}
\left|\psi_{100}^{\mathrm{dr}}\right\rangle= & |100\rangle+K_{13}\left(\frac{|010\rangle}{E-\epsilon_{010}}+\frac{\sqrt{2}|210\rangle}{E-\epsilon_{210}}\right)+K_{23} \frac{|111\rangle}{E-\epsilon_{111}} \\
& +\left(K_{12}+K_{12}^{a}\right) \frac{\sqrt{2}|201\rangle}{E-\epsilon_{201}},
\end{aligned}
$$

where the energies $\epsilon$ of the basis states are only due to noninteracting part $\mathrm{H}_{1}+\mathrm{H}_{2}+\mathrm{H}_{3}$ of Hamiltonian (3). Then from Eq. (22) we obtain

$$
\Omega_{X X}=\frac{2}{\hbar}\left(K_{12}-K_{12}^{a}\right)+\frac{2}{\hbar} K_{13} K_{23}\left(\frac{1}{E-\epsilon_{010}}+\frac{1}{E-\epsilon_{111}}\right) .
$$

For degenerate qubits and weak coupling we can use approximation $E \approx \epsilon_{100}=\epsilon_{001}$ in this equation so that $E-\epsilon_{010}$ $\approx-\hbar\left(\omega_{3}-\omega_{\mathrm{qb}}\right)$ and $E-\epsilon_{111} \approx-\hbar\left(\omega_{3}+\omega_{\mathrm{qb}}\right)$; then using explicit expressions in Eqs. (16)-(18) for the matrix elements we finally obtain

$$
\Omega_{X X}=\frac{\tilde{M}-L_{3}^{*} /\left[1-\left(\omega_{\mathrm{qb}} / \omega_{3}\right)^{2}\right]}{\tilde{L}_{4} \tilde{L}_{5} \omega_{\mathrm{qb}} \sqrt{\widetilde{C}_{1} \widetilde{C}_{2}}}+\frac{C_{a}^{*} \omega_{\mathrm{qb}}}{\sqrt{\tilde{C}_{1} \widetilde{C}_{2}}},
$$

where $L_{3}^{*}=\widetilde{L}_{3}\left(1+\frac{M}{L_{5}}\right)\left(1+\frac{M}{L_{4}}\right), \quad C_{a}^{*}=\widetilde{C}_{a}\left(\widetilde{C}_{1} \widetilde{C}_{2} / C_{1} C_{2}\right)$, and we used $\omega_{3}=\left[\tilde{L}_{3} C_{3}\right]^{-1 / 2}$. Comparing this equation with the classical result Eq. (13), we see that the results coincide under assumptions used for the classical derivation.

Validity of the perturbation theory requires assumptions $\left|K_{13} / \hbar\left(\omega_{3} \pm \omega_{\mathrm{qb}}\right)\right| \ll 1,\left|K_{23} / \hbar\left(\omega_{3} \pm \omega_{\mathrm{qb}}\right)\right| \ll 1,\left|K_{12} / \hbar \omega_{\mathrm{qb}}\right| \ll 1$, and $\left|K_{12}^{a} / \hbar \omega_{\mathrm{qb}}\right| \ll 1$, which basically mean that in Eq. (25) the contributions to $\Omega_{X X}$ due to $\tilde{M}$ and $C_{a}^{*}$ should be much smaller than $\omega_{\mathrm{qb}}$, and the contribution $L_{3}^{*} / \widetilde{L}_{4} \widetilde{L}_{5} \omega_{\mathrm{qb}} \sqrt{\widetilde{C}_{1} \widetilde{C}_{2}}$ should be much smaller than $\left|\omega_{3}-\omega_{\mathrm{qb}}\right|^{2} / \omega_{3}$. Notice that we do not need an assumption $\omega_{\mathrm{qb}} / \omega_{3} \ll 1$, we only need absence of resonance between these frequencies; in fact, $\omega_{3}$ can be even smaller than $\omega_{\mathrm{qb}}$.

To find analytics for $\Omega_{Z Z}$, it is necessary to consider nonlinear oscillators, because this is what we expect from the quasiclassical analysis and also because in the linear case for degenerate qubits $\epsilon_{101}=\epsilon_{200}=\epsilon_{002}$, and therefore there is an ambiguity in defining the logic state $|11\rangle$. In order to calculate $\Omega_{Z Z}$ via Eq. (10), we need to find the eigenenergies $E_{00}$ and $E_{11}$ while calculation of $E_{+}+E_{-} \approx E_{100}^{\mathrm{dr}}+E_{001}^{\mathrm{dr}}$ has been already discussed above. To find the ground-state energy $E_{00}$ we introduce the dressed state $\left|\psi_{000}^{\mathrm{dr}}\right\rangle$ as a state satisfying the Schrödinger equation $\langle n|H| \psi\rangle=E\langle n \mid \psi\rangle$ (for a given $E$ ) for all basis elements $|n\rangle$ except $|000\rangle$ and also satisfying condition $\left\langle 000 \mid \psi_{000}^{\mathrm{dr}}\right\rangle=1$. In a similar way as above, we construct $\left|\psi_{000}^{\mathrm{dr}}\right\rangle$ in the first-order perturbation theory and then find $E_{00}$ as $E_{000}^{\mathrm{dr}}=\left\langle 000|H| \psi_{000}^{\mathrm{dr}}\right\rangle$, using approximation $E \approx \epsilon_{000}$ in the construction of the dressed state. To find the eigenenergy $E_{11}$, we introduce the dressed state $\left|\psi_{101}^{\mathrm{dr}}\right\rangle$ in a similar way, then calculate it in the first order, and then find $E_{11}$ as $E_{101}^{\mathrm{dr}}$ $=\left\langle 101|H| \psi_{101}^{\mathrm{dr}}\right\rangle$ assuming $E \approx \epsilon_{101}$ for the dressed state.
Even though this is a straightforward procedure, now there are infinitely many terms in the first-order dressed states because of the nonlinearity, and there are still many terms even if we keep only lowest orders in nonlinearity. However, most of the contributions to the energies cancel each other in the combination $\hbar \Omega_{Z Z}=E_{000}^{\mathrm{dr}}+E_{101}^{\mathrm{dr}}-E_{100}^{\mathrm{dr}}-E_{001}^{\mathrm{dr}}$ and the largest noncanceling contributions yield

$$
\Omega_{Z Z}=\frac{b_{1} b_{2}}{\hbar}\left[K_{12}+\frac{2 K_{13} K_{23}}{\epsilon_{101}-\epsilon_{111}}\right],
$$

where $b_{i}$ is defined for $i$ th oscillator as

$$
b_{i}=\frac{\left\langle 1\left|\delta \phi_{i}\right| 1\right\rangle-\left\langle 0\left|\delta \phi_{i}\right| 0\right\rangle}{\sqrt{\hbar / 2 m_{i} \omega_{i}}} .
$$

Notice that $\Omega_{Z Z}$ depends on the nonlinearity of qubits (via $b_{1}$ and $b_{2}$ ) while nonlinearity of the coupling junction gives only a small correction (see Appendix) to the second term in Eq. (26), which is neglected in the lowest order. Also notice that in Eq. (26) we neglected terms proportional to $K_{12}^{2}$, because they are on the same order as the neglected terms $\sim\left(K_{13} K_{23}\right)^{2}$. Using the definitions in Eqs. (16)-(18), we rewrite Eq. (26) in the form

$$
\Omega_{\mathrm{ZZ}}=\frac{b_{1} b_{2}}{2} \frac{\tilde{M}-L_{3}^{*}}{\tilde{L}_{4} \tilde{L}_{5} \sqrt{\omega_{1} \omega_{2}} \sqrt{\tilde{C}_{1} \tilde{C}_{2}}},
$$

which coincides with the classical result in Eq. (14) under assumptions used for the classical result, since $\Delta \phi_{10}^{(i)}$ $=b_{i} \widetilde{\Phi}_{0}^{-1} \sqrt{\hbar / 2 \widetilde{C}_{i} \omega_{i}}$.

In deriving Eq. (26) we have used the lowest order of the perturbation theory. However, there are higher-order terms, which are significantly enhanced because the basis state $|101\rangle$ is close to resonance with the states $|200\rangle$ and $|002\rangle$ even for degenerate qubits. Let us account for this effect by analyzing the repulsion between these levels and computing the corresponding shift of the eigenenergy $E_{11}$. Following the above formalism for $\Omega_{X X}$, we find the level splitting due to interaction between $|101\rangle$ and $|200\rangle$ to be $S_{|11\rangle, 20\rangle}$ $=2\left\langle 101\left|H_{\text {int }}\right| \psi_{200}^{\mathrm{dr}}\right\rangle$. Then writing $\left|\psi_{200}^{\mathrm{dr}}\right\rangle$ in the same way as in Eq. (23), we find $S_{|11\rangle,|20\rangle} \approx \sqrt{2} \hbar \Omega_{X X}$, which is essentially the same result as for a qubit interacting with a resonator. ${ }^{28} \mathrm{Be}$ cause of the level repulsion, the eigenenergy $E_{11}$ has a shift by $\left[\epsilon_{200}-\epsilon_{101} \pm \sqrt{\left(\epsilon_{200}-\epsilon_{101}\right)^{2}+2 \hbar^{2} \Omega_{X X}^{2}}\right] / 2$, which in the dispersive case $\epsilon_{101}-\epsilon_{200} \gg\left|\Omega_{X X}\right|$ becomes $\hbar^{2} \Omega_{X X}^{2} / 2\left(\epsilon_{101}-\epsilon_{200}\right)$ (we assume $\epsilon_{101}>\epsilon_{200}$ so the shift is up in energy). In another notation $\epsilon_{101}-\epsilon_{200}=\hbar\left(\omega_{2}-\omega_{1}+\delta \omega_{1}\right)$, where by $\hbar \delta \omega_{i}$ we denote the correction for the second excited level energy, $2 \epsilon_{1}-\epsilon_{0}-\epsilon_{2}$, for $i$ th qubit. A similar shift up in energy for $E_{11}$ comes from the interaction with the level $|002\rangle$. Adding these two contributions, we modify Eq. (28) to become

$$
\begin{aligned}
\Omega_{Z Z}= & \frac{b_{1} b_{2}}{2} \frac{\tilde{M}-L_{3}^{*}}{\tilde{L}_{4} \tilde{L}_{5} \sqrt{\omega_{1} \omega_{2}} \sqrt{\tilde{C}_{1} \tilde{C}_{2}}}+\frac{\Omega_{X X}^{2}}{2} \\
& \times\left(\frac{1}{\omega_{2}-\omega_{1}+\delta \omega_{1}}+\frac{1}{\omega_{1}-\omega_{2}+\delta \omega_{2}}\right) .
\end{aligned}
$$

Notice that for $\Omega_{Z Z}$ we, in general, consider different qubit 
frequencies $\omega_{1}$ and $\omega_{2}$ while in Eq. (25) for $\Omega_{X X}$ we assumed nearly degenerate qubits; however, unless qubit detuning significantly affects $\Omega_{X X}$ (that will be discussed later), we can use definition $\omega_{\mathrm{qb}}=\left(\omega_{1}+\omega_{2}\right) / 2$ in Eq. (25).

Since $\Omega_{Z Z}$ has a major dependence on the qubit nonlinearity, let us discuss it in more detail (see also Appendix). For $i$ th oscillator potential with an additional cubic term, it is convenient to characterize nonlinearity by the ratio $N_{i}$ $=U_{\mathrm{bar}, i} / \hbar \omega_{i, \mathrm{pl}}$, where $U_{\mathrm{bar}, i}$ is the barrier height (assumed to be at $\left.\delta \phi_{j}>0\right)$ so that $N_{i}$ is crudely the number of levels in the quantum well $\left(N_{1,2} \sim 5\right.$ in typical experiments with phase qubits $\left.^{24}\right)$. For a weak cubic nonlinearity $\left(N_{i} \gg 1\right)$ one can derive $^{29}$ the following approximations:

$$
b_{i} \approx 1 / \sqrt{3 N_{i}}, \quad \delta \omega_{i} \approx\left(5 / 36 N_{i}\right) \omega_{i} .
$$

Therefore, away from the point where $\Omega_{X X} \approx 0$, and neglecting corrections due to nonzero $C_{a}, \omega_{\mathrm{qb}} / \omega_{3}$, and $\omega_{2}-\omega_{1}$, the ratio of couplings is

$$
\frac{\Omega_{\mathrm{ZZ}}}{\Omega_{X X}} \approx \frac{1}{6 \sqrt{N_{1} N_{2}}}+\frac{18\left(N_{1}+N_{2}\right)}{5} \frac{\Omega_{X X}}{\omega_{\mathrm{qb}}},
$$

which is quite small for typical experimental parameters. Notice that for $N_{i}=5$ (which is typically used for qubits) the numerical values $b_{i}=0.289$ and $\delta \omega_{i} / \omega_{i}=0.0378$ are significantly different from what is expected from the large- $N$ analytics Eq. (30) (in the cubic approximation for the qubit potential $b_{i}$ and $\delta \omega_{i} / \omega_{i}$ depend only on $N_{i}$ ).

\section{RESIDUAL COUPLING}

Both $\Omega_{X X}$ and $\Omega_{Z Z}$ may cross zero when $L_{3}^{*}$ is varied by adjusting the bias current $I_{B}$. However, they are typically zeroed at different values of $L_{3}^{*}$ that prevents turning the two-qubit coupling completely off. Since $\left|\Omega_{Z Z} / \Omega_{X X}\right| \ll 1$ away from the zero-crossing points, let us characterize the residual coupling by the $Z Z$-coupling value $\Omega_{Z Z}^{\text {res }}$ at the point where $\Omega_{X X}=0$. Using Eqs. (25) and (29), we find for degenerate qubits

$$
\Omega_{\mathrm{ZZ}}^{\mathrm{res}} \approx \frac{b_{1} b_{2}}{2}\left[\frac{\tilde{M}\left(\omega_{\mathrm{qb}} / \omega_{3}\right)^{2}}{\tilde{L}_{4} \tilde{L}_{5} \sqrt{\tilde{C}_{1} \tilde{C}_{2}} \omega_{\mathrm{qb}}}-\frac{C_{a}^{*} \omega_{\mathrm{qb}}}{\sqrt{\tilde{C}_{1} \tilde{C}_{2}}}\right],
$$

where we assumed $\omega_{\mathrm{qb}} / \omega_{3} \ll 1$, as in the experiment.

It is natural to characterize the $\mathrm{OFF} / \mathrm{ON}$ ratio for the adjustable coupling by the ratio $\Omega_{Z Z}^{\text {res }} / \Omega_{X X}^{\mathrm{ON}}$, where $\Omega_{X X}^{\mathrm{ON}}$ is the "fully on" $X X$ coupling. Let us assume the operating regime in which the ON coupling corresponds to zero-bias current, ${ }^{30}$ and at this point $\tilde{M} / L_{3}^{*} \simeq 2$ [see Eq. (25)]. Then $\Omega_{X X}^{\mathrm{ON}}$ $\simeq \tilde{M} / 2 \widetilde{L}_{4} \widetilde{L}_{5} \omega_{\mathrm{qb}} \sqrt{\widetilde{C}_{1} \widetilde{C}_{2}}$, and we find an estimate

$$
\Omega_{\mathrm{ZZ}}^{\mathrm{res}} / \Omega_{X X}^{\mathrm{ON}} \simeq b_{1} b_{2}\left[\left(\omega_{\mathrm{qb}} / \omega_{3}\right)^{2}-C_{a}^{*} \omega_{\mathrm{qb}}^{2} \widetilde{L}_{4} \tilde{L}_{5} / \tilde{M}\right] .
$$

In particular, for $C_{a}=0, N_{1}=N_{2}=5$, and $\omega_{\mathrm{qb}} / \omega_{3} \approx 1 / 5$ (typical experimental parameters), this gives $\mathrm{OFF} / \mathrm{ON} \simeq 3$ $\times 10^{-3}$. The capacitance $C_{a} \simeq M / L_{4} L_{5} \omega_{3}^{2}$ needed to zero the $\mathrm{OFF} / \mathrm{ON}$ ratio is then around $0.6 \mathrm{fF}$ for typical experimental parameters $L_{4,5} \simeq 3 \mathrm{nH}, \quad M \simeq 200 \mathrm{pH}$, and $\omega_{3} / 2 \pi$ $\simeq 30 \mathrm{GHz}$.
Actually, since our analytics is only the leading-order calculation while in $\Omega_{Z Z}^{\text {res }}$ we have an almost exact cancellation of contributions due to $\tilde{M}$ and $L_{3}^{*}$, we cannot expect that Eqs. (32) and (33) are accurate even in the leading order. Nevertheless, we can trust the result that the $\mathrm{OFF} / \mathrm{ON}$ ratio is typically quite small, because both $b_{1} b_{2} \approx\left(3 \sqrt{N_{1} N_{2}}\right)^{-1}$ and the terms in the brackets in Eq. (33) are small. Moreover, the $\mathrm{OFF} / \mathrm{ON}$ ratio can be made exactly zero by choosing proper values for $\omega_{\mathrm{qb}} / \omega_{3}$ and $C_{a}$. Even if our analytics missed a small term in Eq. (33), the $\mathrm{OFF} / \mathrm{ON}$ ratio can be zeroed either by increasing $C_{a}$ or decreasing $\omega_{3}$, since this moves $\Omega_{Z Z}^{\text {res }}$ in the opposite directions.

Since $\Omega_{Z Z}^{\text {res }} / \Omega_{X X}^{\mathrm{ON}}$ is very small or even zero, we have to carefully consider other effects, which do not vanish when both $\Omega_{X X}$ and $\Omega_{Z Z}$ discussed above are zero. One of such effects becomes clear when we consider the case of a strong qubit detuning, $\left|\omega_{1}-\omega_{2}\right| \gg\left|\Omega_{X X}\right|$. In this case one would expect that the eigenstate close to the state $|100\rangle$ should have a negligible contribution of the state $|001\rangle$ and vise versa (so that the logic states $|10\rangle$ and $|01\rangle$ are decoupled); however, actually these contributions cannot be decreased to zero. As seen from Eq. (24), $\Omega_{X X}$ depends on energy $E$, and therefore it is slightly different for the two eigenstates with energies $E_{+}$ and $E_{-}$. The difference $\Delta \Omega_{X X}=\Omega_{X X}^{+}-\Omega_{X X}^{-}$is approximately $-4 K_{13} K_{23}\left(E_{+}-E_{-}\right) / \hbar^{3} \omega_{3}^{2}$, which can be rewritten as

$$
\Delta \Omega_{X X} \approx \frac{-L_{3}^{*}\left|\omega_{1}-\omega_{2}\right|}{\widetilde{L}_{4} \tilde{L}_{5} \omega_{\mathrm{qb}} \sqrt{\widetilde{C}_{1} \widetilde{C}_{2}} \omega_{3}} \simeq-2 \Omega_{X X}^{\mathrm{ON}} \frac{\left|\omega_{1}-\omega_{2}\right|}{\omega_{3}},
$$

where we assumed $\omega_{3} \gg \omega_{1,2}$ and used $L_{3}^{*} \approx \tilde{M}$ for nearly OFF coupling (while $\widetilde{M} / L_{3}^{*} \simeq 2$ in the ON regime at $I_{B}=0$ ). This means that if we zero the amplitude of the state $|100\rangle$ in one of the eigenstates, there will still be a nonzero amplitude of the state $|001\rangle$ in the other eigenstate (in contrast to an ideal two-qubit situation). Choosing the smallest $\Omega_{X X}$ coupling as $\pm \Delta \Omega_{X X} / 2$, we obtain contributions $-\Omega_{X X}^{\mathrm{ON}} / 2 \omega_{3}$ of the wrong states in both eigenstates. So, the error occupation is $\sim\left(\Omega_{X X}^{\mathrm{ON}} / 2 \omega_{3}\right)^{2}$, which for typical parameters is around $10^{-6}$.

Another effect, which is related to inaccuracy of the RWA approximation, can be characterized by the contribution of the state $|101\rangle$ in the ground state. Using the second-order perturbation theory for the dressed state $\left|\psi_{000}^{\mathrm{dr}}\right\rangle$ we find

$$
\left\langle 101 \mid \psi_{000}^{\mathrm{dr}}\right\rangle=\frac{K_{12}+K_{12}^{a}+\frac{K_{13} K_{23}}{\epsilon_{000}-\epsilon_{110}}+\frac{K_{13} K_{23}}{\epsilon_{000}-\epsilon_{011}}}{\epsilon_{000}-\epsilon_{101}},
$$

which is approximately $-\Omega_{X X} / 4 \omega_{\mathrm{qb}}$ when $\Omega_{X X}$ is not close to zero, exactly as expected for the non-RWA contribution $\left(\hbar \Omega_{X X} / 2\right) \sigma_{+}^{(1)} \sigma_{+}^{(2)}$ from the term $\left(\hbar \Omega_{X X} / 2\right) \sigma_{X}^{(1)} \sigma_{X}^{(2)}$ in the twoqubit Hamiltonian. However, we are mostly interested in the case $\Omega_{X X}=0$; then Eq. (35) becomes approximately $-\Omega_{X X}^{\mathrm{ON}} / 2 \omega_{3}$, and the corresponding error occupation is $\sim\left(\Omega_{X X}^{\mathrm{ON}} / 2 \omega_{3}\right)^{2} \simeq 10^{-6}$, same as for the strong-detuning effect.

\section{NUMERICAL RESULTS}

To calculate $\Omega_{X X}$ and $\Omega_{Z Z}$ numerically, we first find the phases $\left\{\phi_{1, \mathrm{st}}, \phi_{2, \mathrm{st}}, \phi_{3, \mathrm{st}}\right\}$, which correspond to the minimum 

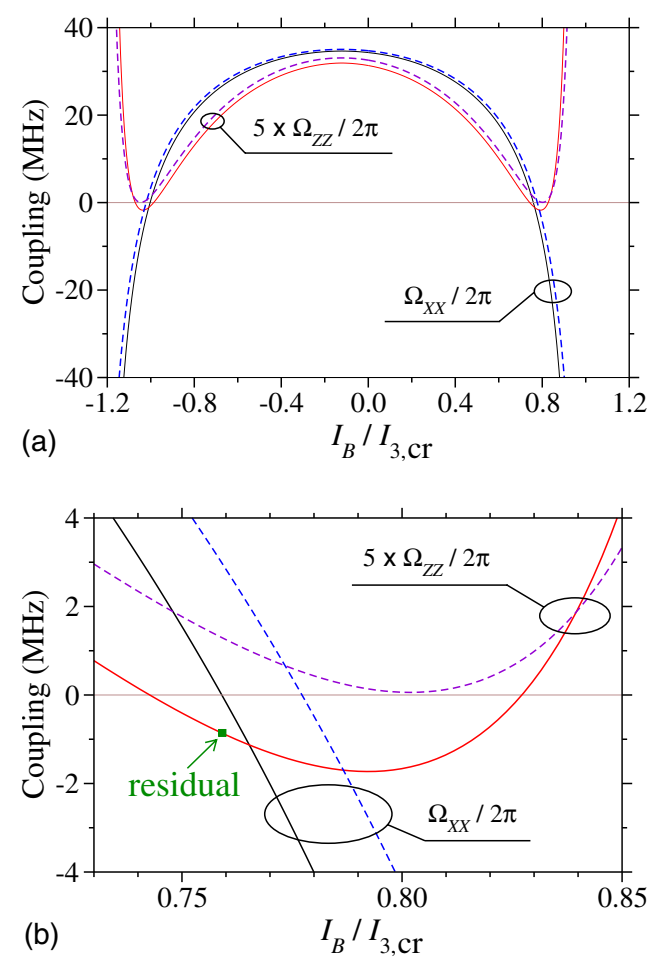

FIG. 2. (Color online) The coupling $\Omega_{X X} / 2 \pi$ and $\Omega_{Z Z} / 2 \pi$ (multiplied by 5 for clarity) as functions of the bias current $I_{B}$. Solid lines show numerical results while dashed lines show analytics using Eqs. (25) and (29). The circuit parameters are: $I_{1, \mathrm{cr}}=I_{2, \mathrm{cr}}$ $=1.5 \mu \mathrm{A}, I_{3, \mathrm{cr}}=3 \mu \mathrm{A}, C_{1}=C_{2}=1 \mathrm{pF}, C_{3}=0.1 \mathrm{pF}, C_{a}=0, L_{1}=L_{2}$ $=0.7 \mathrm{nH}, \quad L_{4}=L_{5}=3 \mathrm{nH}, M=0.2 \mathrm{nH} ; \quad \phi_{e, 4}=\phi_{e, 5}=0, \quad N_{1}=N_{2}=5$ $\left(\omega_{1} / 2 \pi=\omega_{2} / 2 \pi=6.59 \mathrm{GHz}\right)$. The panel (b) is a blow-up of the panel (a) near the crossing points at positive $I_{B}$. We see that $\Omega_{X X}$ $=0$ at $I_{B} / I_{3, \mathrm{cr}}=0.759$ and the residual coupling (square symbol) is $\Omega_{Z Z}^{\text {res }} / 2 \pi=-172 \mathrm{kHz}$.

potential energy in Eq. (1), and then find the eigenfunctions and eigenenergies of Hamiltonian (3)-(5) using the productstate basis of energy levels in the three anharmonic oscillators (in this calculation we use the cubic approximation for the oscillator potentials). After that the coupling $\Omega_{\mathrm{ZZ}}$ is calculated from the eigenenergies using Eq. (10) while for $\Omega_{X X}$ we calculate two values, $\Omega_{X X}^{+}$and $\Omega_{X X}^{-}$, using the definition in Eq. (19). However, for the case of degenerate qubits, which we mostly consider below, there is no difference between $\Omega_{X X}^{+}$and $\Omega_{X X}^{-}$.

Figure 2(a) shows $\Omega_{X X}$ and $\Omega_{Z Z}$ as functions of the bias current $I_{B}$ for the system with the following parameters: ${ }^{30}$ $I_{1, \mathrm{cr}}=I_{2, \mathrm{cr}}=1.5 \mu \mathrm{A}, I_{3, \mathrm{cr}}=3 \mu \mathrm{A}, C_{1}=C_{2}=1 \mathrm{pF}, C_{3}=0.1 \mathrm{pF}$, $C_{a}=0, L_{1}=L_{2}=0.7 \mathrm{nH}, L_{4}=L_{5}=3 \mathrm{nH}$, and $M=0.2 \mathrm{nH}$. We assume $\phi_{e, 4}=\phi_{e, 5}=0$ for the coupler loops while the qubit external fluxes $\phi_{e, 1}=\phi_{e, 2}$ are chosen so that for the qubits $N_{1}=N_{2}=5$; this corresponds to the qubit frequencies $\omega_{1} / 2 \pi$ $=\omega_{2} / 2 \pi=6.59 \mathrm{GHz}$, which are kept constant with changing $I_{B}$ by the compensating change in external fluxes $\phi_{e, 1}$ and $\phi_{e, 2}$. The results for $\Omega_{\mathrm{ZZ}}$ are multiplied by 5 for clarity (to become visually comparable to $\Omega_{X X}$ ). The solid lines in Fig. 2(a) show numerical results while dashed lines show the analytics using Eqs. (25) and (29). One can see that overall the analytics gives a pretty good approximation. Smaller numeri-
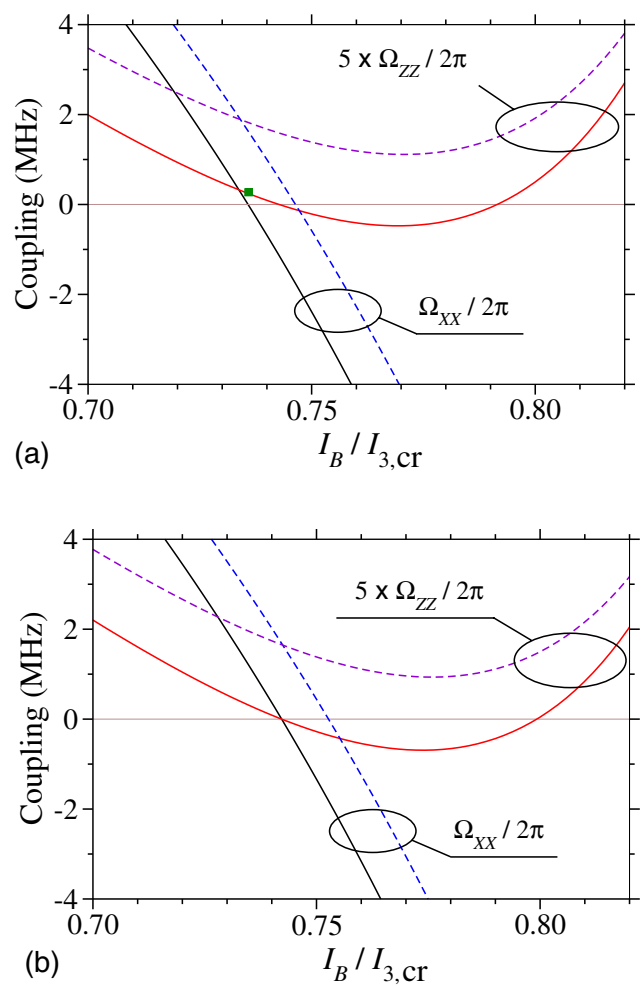

FIG. 3. (Color online) (a) Same as in Fig. 2(b), but for a circuit with $C_{3}=0.3 \mathrm{pF}$. This makes positive $\Omega_{\mathrm{ZZ}}^{\text {res }} / 2 \pi=49 \mathrm{kHz}$ (square symbol). (b) Same as in (a), but with added coupling capacitance $C_{a}=0.155 \mathrm{pF}$, that produces $\Omega_{Z Z}^{\text {res }}=0$, i.e., the couplings $\Omega_{X X}$ and $\Omega_{Z Z}$ are zeroed simultaneously.

cal value for $\Omega_{X X}$ than in analytics can be partially explained by the corrections shown in Eqs. (A7) and (A8) in Appendix. We have checked numerically that the beating frequency of the classical small-amplitude oscillations is close to the analytical quantum result for $\Omega_{X X}$ shown in Fig. 2(a) with a typical difference on the order of $1 \mathrm{MHz}$

The lines in Fig. 2(a) are not symmetric about $I_{B}=0$ because of the current through the coupling junction coming from the qubits, which adds to $I_{B}$ (the curves are symmetric about $I_{B}=-0.122 I_{3, \text { cr }}$; this asymmetry could be removed if the qubits are biased with opposite fluxes, $\phi_{e, 2} \approx-\phi_{e, 1}$, so that the currents from the qubits compensate each other). At zero bias (ON coupling) the coupling $\Omega_{X X} / 2 \pi$ is $34.3 \mathrm{MHz}$, which (analytically) comes from $85.8 \mathrm{MHz}$ coupling due to the mutual inductance $-M$ and compensating $-51.1 \mathrm{MHz}$ from the inductance $\widetilde{L}_{3}$ of the coupling junction (analytical total slightly differs from the numerical result).

At both positive and negative $I_{B}$ the coupling $\Omega_{X X}$ crosses zero because of increase in $\widetilde{L}_{3}$, while $\Omega_{\mathrm{ZZ}}$ barely crosses zero because of the similar increase in $\widetilde{L}_{3}$ and always positive contribution from the level repulsion effect [see Eq. (29)]. Figure 2(b) is a blow-up of Fig. 2(a) near the crossing points at positive $I_{B}$. One can see that numerically calculated residual coupling (at $\Omega_{X X}=0$ ) is $\Omega_{\mathrm{ZZ}}^{\text {res }} / 2 \pi=-172 \mathrm{kHz}$ so that the OFF/ON ratio is $5 \times 10^{-3}$. While this value of $\Omega_{\mathrm{ZZ}}^{\text {res }}$ is on the same order as expected from the analytics (see dashed lines), it has the opposite (negative) sign. This apparently happens because corrections to analytics in this case have a 


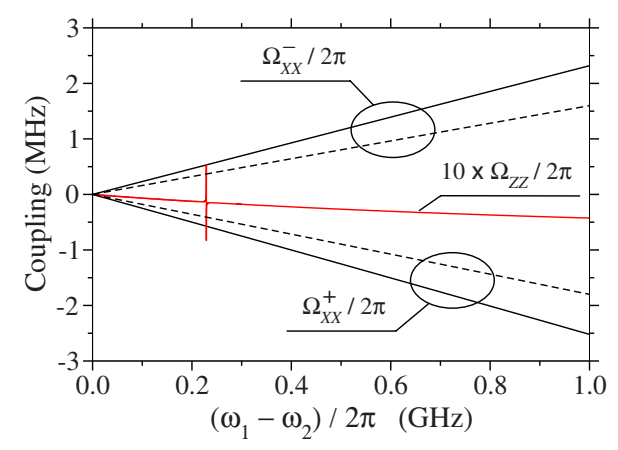

FIG. 4. (Color online) Solid lines: numerical results for $\Omega_{X X}^{+}$, $\Omega_{X X}^{-}$, and $\Omega_{Z Z}$ (multiplied by 10) as functions of the qubit detuning $\omega_{1}-\omega_{2}$ for the parameters of Fig. 3(b) at the point where $\Omega_{X X}$ $=\Omega_{Z Z}=0$. Dashed lines show the analytics for $\Omega_{X X}^{ \pm} \approx \pm \Delta \Omega_{X X} / 2$ from Eq. (34). Error state occupation due to nonzero $\Omega_{X X}^{ \pm}$is 1.5 $\times 10^{-6}$.

stronger effect than the effect of the term $\left(\omega_{\mathrm{qb}} / \omega_{3}\right)^{2}$ in Eq. (25); at the point where $\Omega_{X X}=0$ we have $\omega_{3} / 2 \pi$ $=38.6 \mathrm{GHz}$ so $\left(\omega_{\mathrm{qb}} / \omega_{3}\right)^{2}=0.029$ is really quite small $\left(\omega_{3} / 2 \pi=49.6 \mathrm{GHz}\right.$ at $\left.I_{B}=0\right)$. Notice that a negative value of $\Omega_{Z Z}^{\text {res }}$ makes impossible to zero $\Omega_{Z Z}^{\text {res }}$ by adding the capacitive coupling via $C_{a}$.

In order to make $\Omega_{Z Z}^{\text {res }}$ positive, we can decrease $\omega_{3}$ by increasing the coupling junction capacitance $C_{3}$. Figure 3(a) shows results for the same circuit with $C_{3}=0.3 \mathrm{pF}$, which is three times larger than in Fig. 2. We show only vicinity of the crossing points while the overall shape of the curves is quite close to what is shown in Fig. 2(a); in particular, $\Omega_{X X} / 2 \pi$ $=32.5 \mathrm{MHz}$ at $I_{B}=0$. As we see from Fig. 3(a), now $\Omega_{Z Z}^{\text {res }}$ becomes positive, $\Omega_{Z Z}^{\text {res }} / 2 \pi=49 \mathrm{kHz}$ (at this crossing point $\omega_{3} / 2 \pi=22.9 \mathrm{GHz}$, and it is $28.7 \mathrm{GHz}$ at $\left.I_{B}=0\right)$. The corresponding $\mathrm{OFF} / \mathrm{ON}$ ratio is now $1.5 \times 10^{-3}$.

Obviously, $\Omega_{Z Z}^{\text {res }}=0$ for some intermediate value of $C_{3}$. We have calculated that it happens for $C_{3}=0.253 \mathrm{pF}$ (keeping other parameters unchanged). If $C_{3}$ is larger than this value so that $\Omega_{Z Z}^{\text {res }}$ is positive, we can zero the residual coupling by adding small coupling capacitance $C_{a}$. Figure $3(\mathrm{~b})$ shows the results for $C_{3}=0.3 \mathrm{pF}$ and $C_{a}=0.155 \mathrm{fF}$ (other parameters unchanged), in which case $\Omega_{Z Z}^{\text {res }}=0$.

At the point where $\Omega_{X X}=\Omega_{Z Z}=0$, we have to pay a special attention to other effects which couple the two qubits. In particular, we should consider what happens when the qubits are detuned in frequency. Figure 4 shows $\Omega_{X X}^{+}, \Omega_{X X}^{-}$, and $\Omega_{Z Z}$ (multiplied by 10) as functions of the detuning $\omega_{1}-\omega_{2}$ for the circuit with $C_{3}=0.3 \mathrm{pF}$ and $C_{a}=0.155 \mathrm{fF}$ (other parameters as above) at the bias current $I_{B}=0.742 I_{3, \mathrm{cr}}$; these parameters correspond to the point $\Omega_{X X}=\Omega_{Z Z}=0$ in Fig. 3(b). For the detuning we change the external fluxes $\phi_{e, 1}$ and $\phi_{e, 2}$ (and correspondingly change $N_{1}$ and $N_{2}$ ) while keeping the frequency $\omega_{\mathrm{qb}}=\left(\omega_{1}+\omega_{2}\right) / 2$ unchanged (and we still assume $\left.\phi_{e, 4}=\phi_{e, 5}=0\right)$. As seen in Fig. 4 , the couplings $\Omega_{X X}^{+}$and $\Omega_{X X}^{-}$ coincide when $\omega_{1}=\omega_{2}$; however, their difference grows with the qubit detuning. The dashed lines show the analytics for $\pm \Delta \Omega_{X X} / 2$ using the first expression in Eq. (34). There is a significant difference between the analytical and numerical results because the ratio $\omega_{\mathrm{qb}} / \omega_{3}=6.59 \mathrm{GHz} / 23.0 \mathrm{GHz}$ is not very small; the next order correction to the analytics by the factor $\left[1+3\left(\omega_{\mathrm{qb}} / \omega_{3}\right)^{2}\right]$, which comes from Eq. (24), accounts for most of the difference. Even though $\left|\Omega_{X X}^{ \pm}\right|$grows with the detuning, the corresponding error state occupation $|\alpha / \beta|^{ \pm 2}$ is constant and is only $1.5 \times 10^{-6}$. The detuning also changes $\Omega_{Z Z}$; however, the effect is minor, and $\Omega_{Z Z} / 2 \pi=$ $-42.6 \mathrm{kHz}$ at the detuning of $1 \mathrm{GHz}$. An almost vertical feature on the $\Omega_{Z Z}$ line at the detuning of $0.23 \mathrm{GHz}$ is due to the level crossing between states $|101\rangle$ and $|002\rangle$. It is relatively small because we have chosen the operating point with $\Omega_{X X}=0$ in absence of detuning; otherwise at the level crossing point the coupling $\Omega_{Z Z}$ changes by approximately $\pm \Omega_{X X} / \sqrt{2}$ [see discussion above Eq. (29)].

For the numerical results in this section we have used the cubic approximation for the qubit and coupling oscillator potentials in calculation of the matrix elements of the Hamiltonian. We have also done calculations using the exact potential and checked that the values of $\Omega_{X X}$ and $\Omega_{Z Z}$ change only slightly, though the residual coupling changes more significantly as expected for an almost exact cancellation of contributions with opposite signs.

\section{CONCLUSION}

The main goal of this paper has been calculation of the two-qubit coupling frequencies $\Omega_{X X}$ and $\Omega_{Z Z}$ for the circuit of Fig. 1, and analysis of their dependence on the bias current $I_{B}$, which can be used to turn the coupling on and off. ${ }^{12}$ We have shown that a simple "electrical engineering" analysis for $\Omega_{X X}$ as the beating frequency of two classical oscillators gives a result, Eq. (13), which is close to the analytical (lowest-order) quantum result, Eq. (25) (the formulas coincide, except minor renormalization of parameters). In turn, the quantum analytics for $\Omega_{X X}$ is close to the results of the quantum numerical analysis (Fig. 2); a minor difference is due to higher orders in perturbation. The electrical engineering analysis for $\Omega_{Z Z}$, Eq. (14), is not fully classical; it needs the language of quantum energy levels and shows that $\Omega_{Z Z}$ originates due to anharmonicity of the qubit oscillators, which shifts the average flux. However, this analysis corresponds to only one term in the quantum analytics, Eq. (29), while the other significant contribution is due to the level repulsion between the states with the single excitation in each qubit and with the double excitation in one of them. The results of the numerical quantum analysis for $\Omega_{Z Z}$ are similar to the quantum analytics (Fig. 2).

As expected, our analysis shows that $\Omega_{X X}$ is the main two-qubit coupling in the considered circuit and $\Omega_{Z Z}$ is typically much smaller. Nevertheless, in the analyzed numerical example using realistic experimental parameters, the ratio $\Omega_{X X} / \Omega_{Z Z}$ is only around 5 for the coupling turned on (small $\left.I_{B}\right)$, which means that corrections for nonzero $\Omega_{Z Z}$ in experimental algorithms are necessary.

The most practically important case is when the coupling is almost off. The fact that $\Omega_{X X}$ can be zeroed exactly is rather trivial: a real number changing sign should necessarily cross zero, and $\Omega_{X X}$ obviously changes sign when the effect of the coupling junction inductance $L_{3}$ overcompensated the effect of the magnetic coupling $-M$. The coupling $\Omega_{Z Z}$ does not necessarily change sign because of always positive con- 
tribution from the level repulsion, but $\Omega_{Z Z}$ should be small when $\Omega_{X X}=0$, as follows from the analytical result in Eq. (29). This fact is quite beneficial for the ability to turn the coupling almost off, and we have defined the residual coupling $\Omega_{Z Z}^{\text {res }}$ as the value of $\Omega_{Z Z}$ when $\Omega_{X X}=0$. A natural measure of the coupling OFF/ON ratio is $\Omega_{Z Z}^{\text {res }} / \Omega_{X X}^{\mathrm{ON}}$, where $\Omega_{X X}^{\mathrm{ON}}$ corresponds to the case of small (zero) $I_{B}$. Notice that this ratio depends on the definition in Eq. (9) since we compare different couplings (for example, if the $Z Z$ term was defined in Eq. (9) without the factor 4, then the OFF/ON ratio would decrease four times). As we found from the analytical and numerical calculations, the $\mathrm{OFF} / \mathrm{ON}$ ratio is few times $10^{-3}$ for typical experimental parameters. Most importantly, the $\mathrm{OFF} / \mathrm{ON}$ ratio can be zeroed exactly by properly choosing capacitances $C_{3}$ and/or $C_{a}$.

Even when the above-defined OFF/ON ratio is exactly zero, this does not mean that the qubits can be made completely decoupled. The reason is that the qubits are still physically coupled to the coupling circuit, and the discussion of such effects should go beyond the language of the coupling of logical qubits, which is characterized by only $\Omega_{X X}$ and $\Omega_{Z Z}$. In particular, detuning of the formally decoupled qubits leads to an erroneous state occupation of around $\left(\Omega_{X X}^{\mathrm{ON}} / 2 \omega_{3}\right)^{2}$, which is on the order of $10^{-6}$ for typical experimental parameters. Non-RWA corrections bring errors of the same order.

In this paper we sometimes assumed a high frequency of the coupling oscillator, $\omega_{3} \gg \omega_{\mathrm{qb}}$, as in the experiment. ${ }^{12}$ However, the analytical results in Eqs. (25) and (29), which do not rely on this assumption, show that it is not really needed for the operation of the scheme. Moreover, the adjustable coupling can be even realized for $\omega_{3}<\omega_{\mathrm{qb}}$; however, in that case we have to use positive mutual inductance and we should not expect typically small $\Omega_{Z Z}$ when $\Omega_{X X}=0$.

The calculation of the coupling $\Omega_{X X}$ and $\Omega_{Z Z}$ in this paper is based on the analysis of the eigenfunctions and eigenenergies of the whole system, thus avoiding ambiguity of reducing the whole system to two logical qubits. However, we have also done the analytical calculations by using such reduction. Assuming $\omega_{\mathrm{qb}} / \omega_{3} \ll 1$, we have eliminated the coupling junction degree of freedom by applying the SchriefferWolf transformation and then projecting the resulting Hamiltonian on the coupling junction ground state; after that the Hamiltonian has been truncated to the two-qubit subspace. The obtained results for $\Omega_{X X}$ and $\Omega_{Z Z}$ basically coincide with Eqs. (25) and (28) under the assumptions used.

The analyzed tunable coupler (without $C_{a}$ ) has been realized by Bialczak et al., ${ }^{12}$ and the dependence of the coupling frequency $\Omega_{X X}$ on the bias current $I_{B}$ has been measured experimentally (the coupling $\Omega_{Z Z}$ has not been measured). Due to a relatively small critical current $I_{3, \text { cr }}$ of the coupling junction in the experiment, the coupling $\Omega_{X X}$ is crossing zero at small $I_{B}$ (see Fig. 4d of Ref. 12). Therefore, in contrast to the case shown in our Figs. 2 and 3, the experimental coupler is nearly OFF at $I_{B}=0$. Notice that Fig. $4 \mathrm{~d}$ of Ref. 12 shows $-\Omega_{X X}$ (in our notation), so it increases with $\left|I_{B}\right|$, and the dependence on $I_{B}$ is symmetrized by a horizontal shift. Using the experimental parameters, we have checked that the theoretical result for $\Omega_{X X}$ is quite close to the experimental result (Fig. 4d of Ref. 12 shows a fitting by the simple theory, which is close to the full theory result).
For realization of multiqubit algorithms it is very important that the residual coupling $\Omega_{Z Z}^{\text {res }}$ can be zeroed by proper design of $C_{3}$ and $C_{a}$. If this is not done, the $\mathrm{OFF} / \mathrm{ON}$ ratio is small (few times $10^{-3}$ ), but may still be significant for complicated algorithms. There is a modification of the scheme of Fig. 1, which may further reduce the OFF/ON ratio without using $C_{a}$ and without precise choice of $C_{3}$. The idea is to add blocking capacitors between the qubits and inductors $L_{4,5}$. Then there will be no dc current from the qubits going into the coupling circuit, and this will eliminate the classical interaction effect leading to $\Omega_{Z Z}$ in Eq. (14). Correspondingly, this should eliminate the first term in the quantum result in Eq. (29) for $\Omega_{Z Z}$ so that $\Omega_{Z Z}^{\text {res }}$ should be very small by itself. In the quantum language, this happens because in the modified scheme the capacitive interaction between five oscillators is of the momentum-momentum type (besides one phase-phase interaction due to $M$ ), and the average momentum for any eigenstate of an oscillator is exactly zero. From experimental point of view, the scheme with blocking capacitors is more convenient because it eliminates the need to adjust external fluxes in the qubits when the bias current $I_{B}$ is changed. We have performed preliminary quantum calculations for $\Omega_{X X}$, which confirm that $\Omega_{X X}$ crosses zero when the effective inductance $L_{3}^{\text {eff }}$ compensates the magnetic coupling $-\tilde{M}$, similar to the case without blocking capacitors. However, mathematically this involves compensation of three dozen quantum terms of the same order, so we may expect that the scheme with blocking capacitors is less robust against decoherence than the scheme of Fig. 1. Such a comparative analysis of the schemes with and without blocking capacitors is a subject of further study.

\section{ACKNOWLEDGMENTS}

This work was supported by NSA and IARPA under ARO Grant No. W911NF-08-1-0336.

\section{APPENDIX: CORRECTIONS DUE TO NONLINEARITY}

In this appendix we discuss an oscillator with a weak cubic nonlinearity and show next-order corrections for $\Omega_{X X}$ and $\Omega_{Z Z}$ due to nonlinearity.

Let us consider an oscillator with a cubic nonlinearity, $H=p^{2} / 2 m+\left(m \omega_{\mathrm{pl}}^{2} / 2\right)(\delta \phi)^{2}-\lambda(\delta \phi)^{3}$, where $\omega_{\mathrm{pl}}$ is the plasma frequency and $\lambda>0$ so that there is a finite barrier height $U_{\mathrm{bar}}=m^{3} \omega_{\mathrm{pl}}^{6} / 54 \lambda^{2}$ at positive $\delta \phi$. It is convenient to characterize nonlinearity by the ratio $N=U_{\mathrm{bar}} / \hbar \omega_{\mathrm{pl}}$ so that $N$ is crudely the number of levels in the quantum well.

Nonlinearity changes the eigenstates $|k\rangle$, eigenenergies $\epsilon_{k}=\langle k|H| k\rangle$, and the normalized matrix elements of the coordinate and momentum operators,

$$
c_{k l}=c_{l k}=\frac{\langle k|\delta \phi| l\rangle}{\sqrt{\hbar / 2 m \omega_{\mathrm{pl}}}}, \quad d_{k l}=-d_{l k}=\frac{\langle k|p| l\rangle}{-i \sqrt{\hbar m \omega_{\mathrm{pl}} / 2}} .
$$

For a weak nonlinearity $(N \gg 1)$ one can derive (similar to Ref. 29) the following approximations: 


$$
\begin{gathered}
\frac{\epsilon_{0}}{\hbar \omega_{\mathrm{pl}}}=\frac{1}{2}-\frac{11}{432 N}, \quad \frac{\epsilon_{k}-\epsilon_{k-1}}{\hbar \omega_{\mathrm{pl}}}=1-\frac{5 k}{36 N}, \\
c_{k k}=\frac{2 k+1}{2 \sqrt{3 N}}, \quad c_{01} \approx 1+\frac{0.0509}{N}, \quad c_{02}=\frac{-1}{\sqrt{54 N}}, \\
c_{03}=\frac{0.0170}{N}, \quad c_{12}=\sqrt{2}+\frac{0.144}{N}, \\
d_{k k}=0, \quad d_{01}=1-\frac{0.0880}{N}, \quad d_{02}=-\frac{2}{\sqrt{54 N}},
\end{gathered}
$$

where we keep only the lowest-order nonvanishing corrections and noninteger numbers are numerical results. In particular, these approximations lead to Eq. (30) for $b=\left(c_{11}\right.$ $\left.-c_{00}\right) \sqrt{\omega / \omega_{\mathrm{pl}}}$ and $\delta \omega=\left(2 \epsilon_{1}-\epsilon_{0}-\epsilon_{2}\right) / \hbar$, where $\omega=\left(\epsilon_{1}-\epsilon_{0}\right) / \hbar$. With next-order corrections, Eq. (30) becomes

$$
b \approx \frac{1}{\sqrt{3 N}}+\frac{0.28}{N^{3 / 2}}, \quad \frac{\delta \omega}{\omega} \approx \frac{5}{36 N}+\frac{0.18}{N^{2}} .
$$

Now let us discuss corrections for the analytics for $\Omega_{X X}$ and $\Omega_{Z Z}$, which are next order in nonlinearity while we still use the lowest order in the perturbation theory. This modifies Eq. (24) to become

$$
\begin{aligned}
\Omega_{X X}= & -\frac{2}{\hbar} K_{12}^{a} d_{01,1} d_{01,2}+\frac{2}{\hbar} c_{01,1} c_{01,2}\left\{K_{12}+K_{13} K_{23}\right. \\
& \left.\times\left[\frac{c_{01,3}^{2}}{E-\epsilon_{010}}+\frac{c_{01,3}^{2}}{E-\epsilon_{111}}+\frac{c_{02,3}^{2}}{E-\epsilon_{020}}+\frac{c_{02,3}^{2}}{E-\epsilon_{121}}\right]\right\},
\end{aligned}
$$

where the additional index $i=1,2,3$ in $c_{k l, i}$ and $d_{k l, i}$ is the oscillator number. Now using Eqs. (A2)-(A5) we obtain

$$
\begin{aligned}
\Omega_{X X}= & \frac{1+\frac{0.05}{N_{1}}+\frac{0.05}{N_{2}}}{\tilde{L}_{4} \tilde{L}_{5} \omega_{\mathrm{qb}} \sqrt{\widetilde{C}_{1} \widetilde{C}_{2}}}\left\{\tilde{M}-L_{3}^{*}\left[\frac{1+0.1 / N_{3}}{1-\left(\omega_{\mathrm{qb}} / \omega_{3}\right)^{2}}\right.\right. \\
& \left.\left.+\frac{0.01 / N_{3}}{1-\left(\omega_{\mathrm{qb}} / 2 \omega_{3}\right)^{2}}\right]\right\} \\
& +\frac{\left(1-\frac{0.09}{N_{1}}-\frac{0.09}{N_{2}}\right) \widetilde{C}_{a} \sqrt{\widetilde{C}_{1} \widetilde{C}_{2} \omega_{\mathrm{qb}}}}{C_{1} C_{2}}
\end{aligned}
$$

Equation (26) with account of next-order corrections in nonlinearity becomes

$$
\Omega_{\mathrm{ZZ}}=\frac{b_{1} b_{2}}{\hbar}\left[K_{12}+\frac{2 K_{13} K_{23} c_{01,3}^{2}}{\epsilon_{101}-\epsilon_{111}}+\frac{2 K_{13} K_{23} c_{02,3}^{2}}{\epsilon_{101}-\epsilon_{121}}\right]
$$

that modifies Eq. (28) [and the first term in Eq. (29)] to become

$$
\Omega_{Z Z}=\frac{1+\frac{0.5}{N_{1}}+\frac{0.5}{N_{2}}}{6 \sqrt{N_{1} N_{2}}} \frac{\tilde{M}-L_{3}^{*}\left(1+0.1 / N_{3}\right)}{\tilde{L}_{4} \tilde{L}_{5} \sqrt{\omega_{1} \omega_{2}} \sqrt{C_{1} C_{2}}}
$$

A useful technical trick in deriving these results [as well as Eq. (26)] is to slightly shift the coordinates $\delta \phi_{i}$ for the three oscillators (by slightly changing $\left.\left\{\phi_{1, \mathrm{st}}, \phi_{2, \mathrm{st}}, \phi_{3, \mathrm{st}}\right\}\right)$ to produce $\left\langle 0\left|\delta \phi_{i}\right| 0\right\rangle=0$ for each oscillator; this significantly reduces the number of terms due to nonlinearity in the derivation.

\footnotetext{
*Corresponding author; korotkov@ee.ucr.edu

${ }^{1}$ Yu. A. Pashkin, O. Astafiev, T. Yamamoto, Y. Nakamura, and J. S. Tsai, Quantum Inf. Process. 8, 55 (2009); J. M. Martinis, ibid. 8, 81 (2009); A. A. Houck, J. Koch, M. H. Devoret, S. M. Girvin, and R. J. Schoelkopf, ibid. 8, 105 (2009); R. W. Simmonds, M. S. Allman, F. Altomare, K. Cicak, K. D. Osborn, J. A. Park, M. Sillanpää, A. Sirois, J. A. Strong, and J. D. Whittaker, ibid. 8, 117 (2009); E. Il'ichev, S. H. W. Van der Ploeg, M. Grajcar, and H.-G. Meyer, ibid. 8, 133 (2009); O. Buisson, W. Guichard, F. W. Hekking, L. Levy, B. Pannetier, R. Dolata, A. B. Zorin, N. Didier, A. Fay, E. Hoskinson, Z. H. Peng, I. M. Pop, ibid. 8, 155 (2009); P. M. Echternach, J. F. Schneiderman, M. D. Shaw, and P. Delsing, ibid. 8, 183 (2009); K. Semba, J. Johansson, K. Kakuyanagi, H. Nakano, S. Saito, H. Tanaka, and H. Takayanagi, ibid. 8, 199 (2009); D. A. Bennett, L. Longobardi, V. Patel, W. Chen, D. V. Averin, and J. E. Lukens, ibid. 8, 217 (2009); A. Paila, J. Tuorila, M. Sillanpää, D. Gunnarsson, J. Sarkar, Y. Makhlin, E. Thuneberg, and P. Hakonen, ibid. 8, 245 (2009); W. D. Oliver and S. O. Valenzuela, ibid. 8, 261
}

(2009); D. Vion, A. Aassime, A. Cottet, P. Joyez, H. Pothier, C. Urbina, D. Esteve, and M. H. Devoret, Science 296, 886 (2002); I. Chiorescu, Y. Nakamura, C. J. P. M. Harmans, and J. E. Mooij, ibid. 299, 1869 (2003); A. J. Berkley, H. Xu, R. C. Ramos, M. A. Gubrud, F. W. Strauch, P. R. Johnson, J. R. Anderson, A. J. Dragt, C. J. Lobb, and F. C. Wellstood, ibid. 300, 1548 (2003); T. Duty, D. Gunnarsson, K. Bladh, and P. Delsing, Phys. Rev. B 69, 140503 (2004); B. L. T. Plourde, T. L. Robertson, P. A. Reichardt, T. Hime, S. Linzen, C.-E. Wu, and J. Clarke, ibid. 72, 060506 (2005); P. J. Leek, J. M. Fink, A. Blais, R. Bianchetti, M. Göppl, J. M. Gambetta, D. I. Schuster, L. Frunzio, R. J. Schoelkopf, and A. Wallraff, Science 318, 1889 (2007); S. Han, Y. Yu, X. Chu, S.-I Chu, and Z. Wang, ibid. 293, 1457 (2001); S. Gladchenko, D. Olaya, E. Dupont-Ferrier, B. Douçot, L. B. Ioffe, and M. E. Gershenson, Nat. Phys. 5, 48 (2009).

${ }^{2}$ L. DiCarlo, J. M. Chow, J. M. Gambetta, L. S. Bishop, B. R. Johnson, D. I. Schuster, J. Majer, A. Blais, L. Frunzio, S. M. Girvin, and R. J. Schoelkopf, Nature (London) 460, 240 (2009). 
${ }^{3}$ T. Yamamoto, M. Neeley, E. Lucero, R. Bialczak, J. Kelly, M. Lenander, M. Mariantoni, A. O'Connell, D. Sank, H. Wang, M. Weides, J. Wenner, Y. Yin, A. Cleland, and J. Martinis, arXiv:1006.5084 (unpublished).

${ }^{4}$ M. Neeley, R. Bialczak, M. Lenander, E. Lucero, M. Mariantoni, A. O'Connell, D. Sank, H. Wang, M. Weides, J. Wenner, Y. Yin, T. Yamamoto, A. Cleland, and J. Martinis, arXiv:1004.4246 (unpublished).

${ }^{5}$ L. DiCarlo, M. Reed, L. Sun, B. Johnson, J. Chow, J. Gambetta, L. Frunzio, S. M. Girvin, M. Devoret, and R. Schoelkopf, arXiv:1004.4324 (unpublished).

${ }^{6}$ M. A. Nielsen and I. L. Chuang, Quantum Computation and Quantum Information (Cambridge University Press, Cambridge, 2000).

${ }^{7}$ T. Hime, P. A. Reichardt, B. L. T. Plourde, T. L. Robertson, C. E. Wu, A. V. Ustinov, and J. Clarke, Science 314, 1427 (2006).

${ }^{8}$ A. O. Niskanen, K. Harrabi K. F. Yoshihara, Y. Nakamura, S. Lloyd, and J. S. Tsai, Science 316, 723 (2007).

${ }^{9}$ M. S. Allman, F. Altomare, J. D. Whittaker, K. Cicak, D. Li, A. Sirois, J. Strong, J. D. Teufel, and R. W. Simmonds, Phys. Rev. Lett. 104, 177004 (2010).

${ }^{10}$ R. Harris, A. J. Berkley, M. W. Johnson, P. Bunyk, S. Govorkov, M. C. Thom, S. Uchaikin, A. B. Wilson, J. Chung, E. Holtham, J. D. Biamonte, A. Y. Smirnov, M. H. S. Amin, and A. M. van den Brink, Phys. Rev. Lett. 98, 177001 (2007).

${ }^{11}$ S. H. W. van der Ploeg, A. Izmalkov, A. M. van den Brink, U. Hubner, M. Grajcar, E. Il'ichev, H.-G. Meyer, and A. M. Zagoskin, Phys. Rev. Lett. 98, 057004 (2007).

${ }^{12}$ R. Bialczak, M. Ansmann, M. Hofheinz, M. Lenander, E. Lucero, M. Neeley, A. O'Connell, D. Sank, H. Wang, M. Weides, J. Wenner, T. Yamamoto, A. Cleland, and J. Martinis, arXiv:1007.2219 (unpublished).

${ }^{13}$ A. Blais, A. M. van den Brink, and A. M. Zagoskin, Phys. Rev. Lett. 90, 127901 (2003).

${ }^{14}$ D. V. Averin and C. Bruder, Phys. Rev. Lett. 91, 057003 (2003).

${ }^{15}$ T. V. Filippov, S. K. Tolpygo, J. Mannik, and J. E. Lukens, IEEE Trans. Appl. Supercond. 13, 1005 (2003).

${ }^{16}$ J. Lantz, M. Wallquist, V. S. Shumeiko, and G. Wendin, Phys. Rev. B 70, 140507(R) (2004).

${ }^{17}$ B. L. T. Plourde, J. Zhang, K. B. Whaley, F. K. Wilhelm, T. L.
Robertson, T. Hime, S. Linzen, P. A. Reichardt, C.-E. Wu, and J. Clarke, Phys. Rev. B 70, 140501(R) (2004).

${ }^{18}$ M. Wallquist, J. Lantz, V. S. Shumeiko, and G. Wendin, New J. Phys. 7, 178 (2005).

${ }^{19}$ A. O. Niskanen, Y. Nakamura, and J. S. Tsai, Phys. Rev. B 73, 094506 (2006).

${ }^{20}$ C. Hutter, A. Shnirman, Yu. Makhlin, and G. Schon, Europhys. Lett. 74, 1088 (2006).

${ }^{21}$ A. Galiautdinov and M. Geller, arXiv:quant-ph/0703208 (unpublished).

${ }^{22}$ S. Ashhab, A. O. Niskanen, K. Harrabi, Y. Nakamura, T. Picot, P. C. de Groot, C. J. P. M. Harmans, J. E. Mooij, and F. Nori, Phys. Rev. B 77, 014510 (2008).

${ }^{23}$ B. Peropadre, P. Forn-Diaz, E. Solano, and J. J. Garcia-Ripoll, Phys. Rev. Lett. 105, 023601 (2010).

${ }^{24}$ K. B. Cooper, M. Steffen, R. McDermott, R. W. Simmonds, S. Oh, D. A. Hite, D. P. Pappas, and J. M. Martinis, Phys. Rev. Lett. 93, 180401 (2004).

${ }^{25}$ K. K. Likharev, Dynamics of Josephson Junctions and Circuits (Gordon and Breach, New York, 1986).

${ }^{26}$ A. G. Kofman, Q. Zhang, J. M. Martinis, and A. N. Korotkov, Phys. Rev. B 75, 014524 (2007).

${ }^{27}$ The classical magnetic energy in the coupler is $E_{m}=L_{4} I_{4}^{2} / 2$ $+L_{5} I_{5}^{2} / 2-M I_{4} I_{5}$, where $I_{4}$ and $I_{5}$ are the currents through the inductors $L_{4}$ and $L_{5}$ (flowing in the "down" direction in Fig. 1). When expressed in terms of the fluxes $\Phi_{4}=L_{4} I_{4}-M I_{5}$ and $\Phi_{5}=L_{5} I_{5}-M I_{4}, \quad$ it becomes $E_{m}=\Phi_{4}^{2} / 2 K L_{4}+\Phi_{5}^{2} / 2 K L_{5}$ $+\left(M / K L_{4} L_{5}\right) \Phi_{4} \Phi_{5}$, where $K=1-M^{2} / L_{4} L_{5}$. Notice the sign change in the interaction term, which is important in Eqs. (1) and (14).

${ }^{28}$ A. Blais, R. S. Huang, A. Wallraff, S. M. Girvin, and R. J. Schoelkopf, Phys. Rev. A 69, 062320 (2004).

${ }^{29}$ J. M. Martinis, S. Nam, J. Aumentado, K. M. Lang, and C. Urbina, Phys. Rev. B 67, 094510 (2003).

${ }^{30}$ In the experiment (Ref. 12) the regime of the OFF coupling was actually realized at the bias current $I_{B}$ close to zero. This happened because of designed $M / L_{3} \approx 1$ at $I_{B}=0$. We think that a better mode is when the coupling is OFF at $\left|I_{B}\right| / I_{3, \text { cr }} \lesssim 1$ and consider $M / L_{3} \simeq 2$ at $I_{B}=0$. 